medRxiv preprint doi: https://doi.org/10.1101/2021.07.08.21260171; this version posted July 24, 2021. The copyright holder for this preprint

(which was not certified by peer review) is the author/funder, who has granted medRxiv a license to display the preprint in perpetuity.

it is made available under a CC-BY-NC 4.0 International license.

\title{
1 Simplified point-of-care full SARS-CoV-2 genome sequencing using nanopore technology
}

2 Anton Pembaur ${ }^{1, *}$, Erwan Sallard ${ }^{2} *$, Patrick Philipp Weil ${ }^{1}$, Jennifer Ortelt ${ }^{3}$, Parviz Ahmad-Nejad ${ }^{3}$, Jan

3 Postberg ${ }^{1, \dagger}$

4

5 *both authors contributed equally

6 corresponding author

7

$8{ }^{1}$ Clinical Molecular Genetics and Epigenetics, Faculty of Health, Centre for Biomedical Education \&

9 Research (ZBAF), Witten/Herdecke University, Alfred-Herrhausen-Str. 50, 58448 Witten, Germany

$10{ }^{2}$ Institute of Virology and Microbiology, Faculty of Health, Centre for Biomedical Education \&

11 Research (ZBAF), Witten/Herdecke University, Stockumer Str. 10, 58453 Witten, Germany

$12{ }^{3}$ HELIOS University Hospital Wuppertal, Institute of Medical Laboratory Diagnostics, Centre for

13 Clinical \& Translational Research (CCTR), Witten/Herdecke University, Heusnerstr. 40, 42283

14 Wuppertal, Germany

16 E-Mail addresses:

17 Anton Pembaur:

anton.pembaur@uni-wh.de

18 Erwan Sallard:

erwan.sallard@uni-wh.de

19 Patrick Philipp Weil:

patrick.weil@uni-wh.de

20 Jennifer Ortelt:

jennifer.ortelt@helios-gesundheit.de

21 Parviz Ahmad-Nejad:

parviz.ahmad-nejad@helios-gesundheit.de

22 Jan Postberg:

jan.postberg@uni-wh.de 
medRxiv preprint doi: https://doi.org/10.1101/2021.07.08.21260171; this version posted July 24, 2021. The copyright holder for this preprint

(which was not certified by peer review) is the author/funder, who has granted medRxiv a license to display the preprint in perpetuity.

It is made available under a CC-BY-NC 4.0 International license.

\section{Abstract}

35 Background: The scale of the ongoing SARS-CoV-2 pandemic warrants the urgent establishment of a

36 global decentralized surveillance system to recognize local outbreaks and the emergence of novel

37 variants-of-concern. Among available deep-sequencing technologies, nanopore-sequencing could be

38 an important cornerstone, since it is mobile, scalable and costs-effective. Therefore, streamlined

39 nanopore-sequencing protocols need to be developed and optimized for SARS-CoV-2 variants

40 identification.

41 Results: We adapted and simplified existing workflows using the 'midnight' 1,200 bp amplicon split

42 primer sets for PCR, which produce tiled overlapping amplicons covering almost the entire SARS-

43 CoV-2 genome. Subsequently, we applied Oxford Nanopore Rapid Barcoding and the portable

44 MinION Mk1C sequencer combined with the interARTIC bioinformatics pipeline. We tested a

45 simplified and less time-consuming workflow using SARS-CoV-2-positive specimens from clinical

46 routine and identified pre-analytical parameters, which may help to decrease sequencing failures rates.

47 Complete pipeline duration was approx. $7 \mathrm{hrs}$ for one specimen and approx. $11 \mathrm{hrs}$ for 12 multiplexed

48 barcoded specimens.

49 Conclusions: The adapted protocol contains less processing steps and can be completely conducted

50 within one working-day. Diagnostic CT values are principal criteria for specimen selection.

\section{Keywords}

$53(+)$ RNA genome sequencing, COVID-19 surveillance, variant-of-concern (VOC)

\section{Background}

56 To face the ongoing SARS-CoV-2 pandemic, a global decentralized warning system is being

57 established to recognize local outbreaks and the emergence of novel variants-of-concern (VOC). A

58 particular focus is given to the identification of VOCs with accelerated transmission rates, increased

59 infectivity or immune escape mutations, since these variants would warrant adaptations in containment

60 and vaccination strategies (Cobey, Larremore et al. 2021, Gupta 2021, Harvey, Carabelli et al. 2021,

61 Winger and Caspari 2021). Moreover, the search for the zoonotic origin of SARS-CoV-2 from

62 comparative analyses of genomic data is an ongoing issue with relevance for the early recognition of

63 future outbreak scenarios (Andersen, Rambaut et al. 2020, V'Kovski, Kzel et al. 2021).

64 Among the available deep-sequencing technologies, nanopore-sequencing could be an important cornerstone, since it is mobile, scalable and acquisition investments are comparably low. Further,

66 nanopore sequencing devices do not require large-scale IT infrastructure. In the past they were already

67 involved actions of genome surveillance, e.g. the 2015 Ebola outbreak in Liberia, Guinea and sierra

68 Leone (Quick, Loman et al. 2016), and since then there has been a substantial technological progress.

69 However, at least for smaller hospital laboratories with lower throughput, it is desirable to develop

70 protocols as streamlined as possible. 
medRxiv preprint doi: https://doi.org/10.1101/2021.07.08.21260171; this version posted July 24, 2021. The copyright holder for this preprint

(which was not certified by peer review) is the author/funder, who has granted medRxiv a license to display the preprint in perpetuity. It is made available under a CC-BY-NC 4.0 International license .

71 Nanopore sequencing allows the sequencing of either DNA or RNA (Garalde, Snell et al. 2018), and

72 does not require PCR amplification. Furthermore the technique has the potential of producing very

73 long, continuous reads, which theoretically allows to sequence in only one read the 29.903nt long

74 (+)RNA genome of SARS-CoV-2 (Taiaroa, Rawlinson et al. 2020), or its deriving cDNA after reverse 75 transcription (Figure 1).

76 With the aims of facilitating implementation in routine diagnostics with lower specimens throughput

77 and of simplifying the workflow, we tested modifications of existing ARTIC protocols for SARS-

78 CoV-2 full length (+)RNA genome sequencing (Freed, Vlkova et al. 2020, Li, Wang et al. 2020,

79 Tyson, James et al. 2020). Furthermore, we tested the simplified and less time-consuming workflow

80 on confirmed SARS-CoV-2-positive specimens from clinical routine and identified pre-analytical

81 parameters, which may help to decrease the rate of sequencing failures.

\section{Methods}

Nucleic acids isolation

Specimens included nasopharyngeal swabs (Xebios Diagnostics), which underwent routine COVID-19 diagnostic testing. For the collection and use after routine diagnostics procedures we obtained approval of the Witten/Herdecke University Ethics board (No. 160/2020 [For more details, see 'declarations' below.]). RNA purification was performed via magnetic beads (Seegene NIMBUS/Tanbead). Alternatively, total RNA was purified from $250 \mu \mathrm{L}$ liquid specimen using $750 \mu \mathrm{L}$ QIAzol lysis reagent (Qiagen, Cat. No. 158845) upon manufacturer's recommendations, or using silica columns (QiaAmp Viral Mini Kit, Qiagen).

RT-PCR, quality assessments and library preparation

The 'midnight' split primer set from the ARTIC protocol was used for SARS-CoV-2 cDNA amplification in 2 multiplex PCR reactions. To avoid overlaps during multiplex PCR, each single-tube PCR reaction generates consecutively tiled, non-overlapping 1,200 bp amplicons. Mixed together after PCR, both resulting complementary amplicon mixtures cover almost the entire SARS-CoV-2 genome

99 (Freed, Vlkova et al. 2020).

100 Combined reverse transcription and amplification of multiple 1,200 bp amplicons (RT-PCR) was performed in single tube $20 \mu \mathrm{L}$ reactions using the Luna One-Step RT-qPCR Kit (NEB; E3005). For RT-PCR, $8 \mu \mathrm{L}$ of purified template RNA were used for each reaction. From $100 \mu \mathrm{M}$ primer pools, 1 $\mu \mathrm{L}$ was used in each reaction. Reverse transcription was performed at $55^{\circ} \mathrm{C}$ for 30 minutes, followed

104 by incubation at $95^{\circ} \mathrm{C}$ for one minute. Then 34 cycles (pool 1) or 30 cycles (pool 2) of denaturation at $10595^{\circ} \mathrm{C}$ for 20 seconds and annealing and extension in one step at $60^{\circ} \mathrm{C}$ for 210 seconds were performed.

106 A final extension was performed at $65^{\circ} \mathrm{C}$. During the implementation phase amplicon sizes and DNA

107 concentrations were routinely checked by agarose gels or by microvolume electrophoresis (Agilent 
medRxiv preprint doi: https://doi.org/10.1101/2021.07.08.21260171; this version posted July 24, 2021. The copyright holder for this preprint

(which was not certified by peer review) is the author/funder, who has granted medRxiv a license to display the preprint in perpetuity.

It is made available under a CC-BY-NC 4.0 International license .

108 Bioanalyzer, Agilent DNA 12000 kit). Thereafter, amplicons from primer pools 1 and 2 were

109 quantified by fluorimetry (Promega Quantus) and then mixed at equal concentrations. Library

110 preparation was done using the Rapid Barcoding Sequencing Kit (Oxford nanopore; SQK-RBK004)

111 upon manufacturers recommendations.

112

113 Nanopore sequencing

114 Sequencing was performed on a MinION Mk1C with the options 'basecalling' and 'demultiplexing'

115 being enabled. As output format, FAST5 and FASTQ files were chosen. Sequencing time was set for

11672 hours as default. Sequencing was stopped after reaching at least 10 megabases for each barcode.

118 Bioinformatics

119 Consensus sequences were built from the barcode-sorted, quality-filtered FAST5 and FASTQ files

120 containing sequencing reads, using the interARTIC pipeline. Except otherwise stated, the

121 'Nanopolish' algorithm was routinely used.

122 Installation and usage of interARTIC pipeline was done following the developers' instructions:

123 https://psy-fer.github.io/interARTIC/installation/. For faster analysis, all available threads were

124 activated in the advanced settings. The interARTIC pipeline performs read filtering, alignments and

125 returns a consensus FASTA file as well as coverage charts for visualization (Figure 2B-H).

126 Consensus FASTA files were uploaded to the Nextstrain webapp (https://clades.nextstrain.org) to

127 perform phylogenetic analyses (Hadfield, Megill et al. 2018).

129 Data availability

130 FASTQ files and assembled FASTA formatted consensus sequences are available

131 (https://t1p.de/minion-seqdata)

134 Results and Discussion

135 We defined milestones to simplify the protocol and decrease hands-on sample time and working step

136 numbers. 1 . We tested whether specimens can be directly taken from residual diagnostic specimens

137 extracted from 96-deepwell-plates using magnetic beads (Seegene NIMBUS/Tanbead). For

138 comparison we applied RNA purification protocols using silica columns (QiaAmp Viral Mini Kit,

139 Qiagen) or guanidinium isothiocyanate (GITC) for RNA extraction (QIAzol lysis reagent, Qiagen). 2.

140 We tested whether reverse transcription can be successfully primed by SARS-CoV-2-specific primers,

141 which are subsequently used for multiplex 1,200 bp amplicon amplification. Purified RNA from the

142 different extraction protocols of milestone 1 was used for cDNA synthesis and successive multiplex

143 PCR with the 2 'midnight' split 1,200 bp amplicon primer pools in single tube reactions 3 . We aimed

144 at improving the efficiency of the nanopore sequencing workflow by using the onboard Guppy 
medRxiv preprint doi: https://doi.org/10.1101/2021.07.08.21260171; this version posted July 24, 2021. The copyright holder for this preprint

(which was not certified by peer review) is the author/funder, who has granted medRxiv a license to display the preprint in perpetuity.

It is made available under a CC-BY-NC 4.0 International license.

145 basecalling capability of the Oxford Nanopore MinION Mk1C device. Moreover, implementation of

146 the interARTIC interface should help to avoid command line-based bioinformatics analyses as much

147 as possible in order to provide a user-friendly and efficient analysis pipeline.

149 Protocol implementation considering differences in viral loads and influences of RNA extraction

150 protocols

151 Sequencing was performed from serially diluted specimens of purified RNA from patients' samples,

152 which exhibited low cycle threshold $(\mathrm{CT})$ value $(\mathrm{CT}=16-18)$ after routine diagnostics $\mathrm{RT}-\mathrm{qPCR}(\mathrm{N}$

153 gene, RdRP gene; Seegene). Using the quantitative reference sample Ch07470 for calibration we

154 determined that a $\mathrm{CT}=25$ (respectively 16 and 18 ) corresponded to a SARS-CoV-2 copy number of

$1551.0 \times 10^{6}$ (respectively $5.12 \times 10^{8}$ and $1.28 \times 10^{8}$ ). Routinely used dilution factors were $2^{0}-2^{-10}$ in order to

156 cover several CT value magnitudes and to simulate different amounts of viral loads. In terms of RNA

157 yield and 1,200 bp amplicon PCR performance the magnetic beads-based RNA purification protocol

158 outperformed slightly the GITC method as well as the column-based protocol, but we did not observe

159 differences in read and coverage quality between these different isolation methods (Figure S1). Since

160 direct sampling from 96-deepwell plates allowed us to directly exploit residual specimens, which

161 remained after routine RT-qPCR diagnostics, we decided to focus on magnetic beads-based RNA

162 purification during further protocol development. Moreover, it shortened and simplified the workflow.

163 After semiquantitative or quantitative RT-PCR using multiplex primer pools 1 and 2 in separate single

164 tube reactions for combined reverse transcription of the SARS-CoV-2 (+)RNA and amplification of

$1651,200 \mathrm{bp}$ amplicons, band intensities exhibited strong dependence on viral loads. Moreover, after 32

166 PCR cycles band intensities using primer pool 1 were weaker when compared with primer pool 2

167 (Figure S2). We determined that 34 PCR cycles for primer pool 1 and 30 cycles for primer pool 2 were

168 a good compromise. Therefore, reverse transcription can be successfully primed by the 'midnight'

169 primers.

171 Surveillance of multiplex nanopore sequencing and multiple reuses of flow cells

172 Using the Rapid Barcoding Kit (SQK-RBK004, Oxford Nanopore) enabled us to barcode the PCR

173 products without purification steps and sequence them immediatley. As a standard, we used 12

174 barcoded libraries for multiplex sequencing on R9 flow cells. In contrast to the Oxford Nanopore

175 MinION Mk1B, the MinION Mk1C device features onboard guppy basecalling. In combination with

176 the Rapid Barcoding Kit, we exploited this opportunity for real-time surveillance of basecalling and

177 demultiplexing for each of the 12 multiplexed samples per run. This enabled us to recognize the exact

178 time point at which a reading depth of approx. $10 \mathrm{Mbp}$ per barcode was achieved. The time to reach

179 this threshold depended heavily on the viral load (simulated by serially diluted samples). Decreased

180 viral loads let to a considerable decrease of passed reads (Figure 2A). A manual stop of sequencing

181 followed by flow cell washing (Flow Cell Wash Kit, EXP-WSH004, Oxford Nanopore) allowed us to 
medRxiv preprint doi: https://doi.org/10.1101/2021.07.08.21260171; this version posted July 24, 2021. The copyright holder for this preprint

(which was not certified by peer review) is the author/funder, who has granted medRxiv a license to display the preprint in perpetuity.

it is made available under a CC-BY-NC 4.0 International license.

182

183

184

185

186

187

188

189

190

191

192

193

194

195

196

197

198

199

200

201

202

203

204

205

206

207

208

209

210

211

212

213

214

215

216

217

218

reuse a single flow cell for a series of 3 sequencing runs, each using 12 multiplexed barcoded libraries.

This specific scenario resulted in costs of approx. 40 USD per sample. Theoretically, the possible number of reuses depends largely on the duration of the sequencing, which itself depends mainly on the number of used barcodes. Further, if a MinION Mk1B is considered for this purpose, a similar surveillance functionality could be achieved using RAMPART (https://github.com/articnetwork/rampart) on a dedicated LINUX environment.

\section{Assembly of full SARS-CoV-2 genomes and pathogen genome data analyses}

For mapping and full length SARS-CoV-2 genome assembly, we used the FASTQ files resulting from Guppy basecalling in order to generate FASTA formatted consensus sequence files. We used the ARTIC pipeline through a graphical user interface (interARTIC; $\underline{\text { https://github.com/Psy- }}$ Fer/interARTIC). Once installed, this is an easy to use and relatively fast pipeline with only five minutes hands-on time, which enables the use of the 'Nanopolish' or 'Medaka' algorithms for simultaneous analyses of multiplexed barcoded samples. As an example, Figure 2B shows the complete and deep coverage of the complete SARS-CoV-2 genome after the combination of pools 1 (light blue) and 2 (pink). This demonstrates that all contained 1,200 bp amplicon were specifically and efficiently amplified during the combined RT-PCR reaction (Figure 2B).

To compare the interARTIC and Geneious Prime pipelines, we used exactly the same FASTQ file from the same sample shown in Figure 2B (https://go.geneious.com/video/how-to-assemblecoronavirus-genomes). Phylogenetic analyses with Nextstrain using the FASTA consensus files obtained from the interARTIC or the Geneious Prime pipelines resulted in considerably different phylogenetic distances in clade 20I, showing that the bioinformatics pipeline influences the result (Figure S3). The interARTIC pipeline proved superior in terms of coverage and sequencing depth. Notably, within the interARTIC pipeline both options, the 'Nanopolish' and 'Medaka' algorithms performed equally well with respect to consensus sequence quality, but 'Medaka' was considerably faster.

We observed that the consensus sequences returned by the 'Nanopolish' and 'Medaka' algorithms contain numerous unsolved regions (for which only 'N's are indicated). Interestingly, the regions unsolved by one algorithm were generally solved by the other, so we assumed that the two consensus sequences could be combined to produce a 'super-consensus' with improved variant prediction value.

2 We developed a Python code that merges the 'Nanopolish' and 'Medaka' consensus sequences and generates the corresponding variant calling file. As expected, the 'super-consensus' contained less unsolved regions when compared with the 'Nanopolish' and 'Medaka' consensus sequences alone (Figure 4B). In addition, it retained the high quality mutations, which were identified by both algorithms, while removing most false-positives probably caused by sequencing and alignment errors

17 (Figure 4A). Consequently, our consensus-merging code improves the quality of variant calling and 8 highlights the complementarity of 'Nanopolish' and 'Medaka' for nanopore-sequencing of SARS- 
medRxiv preprint doi: https://doi.org/10.1101/2021.07.08.21260171; this version posted July 24, 2021. The copyright holder for this preprint

(which was not certified by peer review) is the author/funder, who has granted medRxiv a license to display the preprint in perpetuity.

It is made available under a CC-BY-NC 4.0 International license.

219 CoV-2 and others. Nevertheless, this quality increase comes at the cost of information loss such as the

220 number of reads per variant or other metadata which were initially generated by 'Nanopolish' and

221 'Medaka' and are not transferred to the 'super-consensus'.

222 Serial input RNA dilutions or, respectively, viral load influenced the depth of sequencing (Figure 2C-

223 H). For the output of high-quality consensus sequences in the FASTA file format a coverage threshold

224 of 20 was used as default. We generally observed that this could be reached when a SARS-CoV-2 titer

225 of $4 \times 10^{6}$ was given. Viral copy numbers lower than $4 \times 10^{6}$ were associated with incompletely

226 assembled SARS-CoV-2 genomes. Thus, we provide here a convincing line of evidence that the copy

227 number-normalized CT values of diagnostic RT-qPCR can be used as the criterion of sequencing

228 success.

229 Single or batch high-quality consensus FASTA formatted sequences were used for phylogenetic tree

230 visualization and variant calling using the Nextstrain webapp (https://clades.nextstrain.org) (Hadfield,

231 Megill et al. 2018). In our hands, the obtained sequences could faithfully be assigned to specific clades

232 in the reference tree (Figure 3). Again, an influence of viral load was observed. However, despite

233 incomplete coverage in those cases enough informative sequence data could be obtained for

234 phylogenetic analyses from several low copy number samples. As a result of serial sample dilutions,

235 we observed deviating phylogenetic distances within the clade, wherein specimens classification

236 occurred (Figure 3A), which eventually could lead to incorrect clade association. The use of

237 specimens from diagnostic routine with viral copy numbers higher than approx. 4x $10^{6}$ apparently led

238 to their faithful association with different clades, which were clades 20I and 19A in the shown

239 example (Figure 3B). The 'Nanopolish' and 'Medaka' consensus as well as the merged super-

240 consensus could be reliably associated to the corresponding clade (20I in the shown example). The

241 'Medaka' consensus mapped at a greater distance than the 'Nanopolish' consensus, probably because

242 'Medaka' algorithm does not correct frameshifts, while the super-consensus had an intermediary

243 distance between the two other consensus (Figure 4C).

245 Taken together, the main achievements of an optimized workflow are: 1. Purified RNA from SARS-

246 CoV-2-positive patients can be directly taken from residual diagnostic specimens in 96-deepwell-

247 plates; 2. cDNA synthesis and successive multiplex PCR with 2 split primer pools can be performed in

248 single tube reactions. Since cDNA synthesis is primed by SARS-CoV-2-specific primers for 1,200 bp

249 amplicon amplification, there is no need for use of unspecific hexanucleotide priming. 3. Onboard

250 Guppy basecalling with the Oxford Nanopore MinION Mk1C device and implementation of the

251 interARTIC led to a further reduction of working steps and hands-on time (Figure 5). Implementation

252 in smaller hospital laboratories with lower specimens' throughput can be easily done at moderate

253 costs. 
medRxiv preprint doi: https://doi.org/10.1101/2021.07.08.21260171; this version posted July 24, 2021. The copyright holder for this preprint (which was not certified by peer review) is the author/funder, who has granted medRxiv a license to display the preprint in perpetuity. It is made available under a CC-BY-NC 4.0 International license .

255 We provide a detailed protocol for all steps here, which includes the Python code (Supplementary

256 Information 1) and corresponding command line: dx.doi.org/10.17504/protocols.io.bwhppb5n

\section{Conclusions}

260 The adapted protocol contains less processing steps. Diagnostic CT values are the principal criteria for specimen selection. After diagnostic qRT-PCR, multiplex library preparation, quality controls, nanopore sequencing and the bioinformatic pipeline can be completely conducted within one working-

263 day.

\section{List of abbreviations}

$266(+)$ RNA (sense polarity [single-stranded] ribonucleic acid), RdRP (RNA-dependent RNA polymerase), RT-qPCR (reverse transcription-quantitative polymerase chain reaction, SARS-CoV-2 (severe acute respiratory syndrome coronavirus 2)

\section{Declarations}

\section{Ethics approval and consent to participate}

272 For the collection and use of specimens from clinical routine at Helios University Hospital Wuppertal

273 (North Rhine-Westphalia, Western Germany) we obtained approval of the Witten/Herdecke University

274 Ethics board (No. 160/2020). The positive ethics vote included the use of routinely sampled and

275 SARS-CoV-2-confirmed specimens, which underwent COVID-19 RT-qPCR diagnostics procedures

276 even for cases, where informed written consent could not be asked for. When possible, we obtained

277 informed written consent from hospitalized patients or legal guardians. All work has been conducted

278 according to the principles expressed in the Declaration of Helsinki.

\section{Competing interests}

281 There are no conflicting interests for none of the authors, which need declaration.

\section{Funding}

284 The study was financed by own institutional means.

\section{Authors' contributions}

287 AP prepared libraries for nanopore-seq and conducted the sequencing. PAN and JO collected

288 specimens and conducted diagnostic SARS-CoV-2 qPCR. AP and PPW performed nucleic acids

289 quality controls. AP, ES and JP contributed to the bioinformatics analyses. ES developed the Python

290 code. PAN and JP designed the sudy. AP, ES, PPW and JP wrote the paper. 
medRxiv preprint doi: https://doi.org/10.1101/2021.07.08.21260171; this version posted July 24, 2021. The copyright holder for this preprint (which was not certified by peer review) is the author/funder, who has granted medRxiv a license to display the preprint in perpetuity. It is made available under a CC-BY-NC 4.0 International license .

\section{References}

Andersen, K. G., A. Rambaut, W. I. Lipkin, E. C. Holmes and R. F. Garry (2020). "The proximal origin of SARS-CoV-2." Nat Med 26(4): 450-452.

Cobey, S., D. B. Larremore, Y. H. Grad and M. Lipsitch (2021). "Concerns about SARS-CoV-2 evolution should not hold back efforts to expand vaccination." Nat Rev Immunol 21(5): 330-335. Freed, N. E., M. Vlkova, M. B. Faisal and O. K. Silander (2020). "Rapid and inexpensive wholegenome sequencing of SARS-CoV-2 using $1200 \mathrm{bp}$ tiled amplicons and Oxford Nanopore Rapid Barcoding." Biol Methods Protoc 5(1): bpaa014.

Garalde, D. R., E. A. Snell, D. Jachimowicz, B. Sipos, J. H. Lloyd, M. Bruce, N. Pantic, T. Admassu, P. James, A. Warland, M. Jordan, J. Ciccone, S. Serra, J. Keenan, S. Martin, L. McNeill, E. J. Wallace, L. Jayasinghe, C. Wright, J. Blasco, S. Young, D. Brocklebank, S. Juul, J. Clarke, A. J. Heron and D. J. Turner (2018). "Highly parallel direct RNA sequencing on an array of nanopores." Nat Methods 15(3): 201-206.

Gupta, R. K. (2021). "Will SARS-CoV-2 variants of concern affect the promise of vaccines?" Nat Rev Immunol 21(6): 340-341.

Hadfield, J., C. Megill, S. M. Bell, J. Huddleston, B. Potter, C. Callender, P. Sagulenko, T. Bedford and R. A. Neher (2018). "Nextstrain: real-time tracking of pathogen evolution." Bioinformatics 34(23): 4121-4123.

Harvey, W. T., A. M. Carabelli, B. Jackson, R. K. Gupta, E. C. Thomson, E. M. Harrison, C. Ludden, R. Reeve, A. Rambaut, C.-G. U. Consortium, S. J. Peacock and D. L. Robertson (2021). "SARS-CoV2 variants, spike mutations and immune escape." Nat Rev Microbiol 19(7): 409-424.

Li, J., H. Wang, L. Mao, H. Yu, X. Yu, Z. Sun, X. Qian, S. Cheng, S. Chen, J. Chen, J. Pan, J. Shi and X. Wang (2020). "Rapid genomic characterization of SARS-CoV-2 viruses from clinical specimens using nanopore sequencing." Sci Rep 10(1): 17492.

Quick, J., N. J. Loman, S. Duraffour, J. T. Simpson, E. Severi, L. Cowley, J. A. Bore, R. Koundouno, G. Dudas, A. Mikhail, N. Ouedraogo, B. Afrough, A. Bah, J. H. Baum, B. Becker-Ziaja, J. P. Boettcher, M. Cabeza-Cabrerizo, A. Camino-Sanchez, L. L. Carter, J. Doerrbecker, T. Enkirch, I. G. G. Dorival, N. Hetzelt, J. Hinzmann, T. Holm, L. E. Kafetzopoulou, M. Koropogui, A. Kosgey, E. Kuisma, C. H. Logue, A. Mazzarelli, S. Meisel, M. Mertens, J. Michel, D. Ngabo, K. Nitzsche, E. Pallash, L. V. Patrono, J. Portmann, J. G. Repits, N. Y. Rickett, A. Sachse, K. Singethan, I. Vitoriano, R. L. Yemanaberhan, E. G. Zekeng, R. Trina, A. Bello, A. A. Sall, O. Faye, O. Faye, N. Magassouba, C. V. Williams, V. Amburgey, L. Winona, E. Davis, J. Gerlach, F. Washington, V. Monteil, M. Jourdain, M. Bererd, A. Camara, H. Somlare, A. Camara, M. Gerard, G. Bado, B. Baillet, D. Delaune, K. Y. Nebie, A. Diarra, Y. Savane, R. B. Pallawo, G. J. Gutierrez, N. Milhano, I. Roger, C. J. Williams, F. Yattara, K. Lewandowski, J. Taylor, P. Rachwal, D. Turner, G. Pollakis, J. A. Hiscox, D. A. Matthews, M. K. O'Shea, A. M. Johnston, D. Wilson, E. Hutley, E. Smit, A. Di Caro, R. Woelfel, K. Stoecker, E. Fleischmann, M. Gabriel, S. A. Weller, L. Koivogui, B. Diallo, S. Keita, A. Rambaut, P. Formenty, S. Gunther and M. W. Carroll (2016). "Real-time, portable genome sequencing for Ebola surveillance." Nature 530(7589): 228-232.

Taiaroa, G., D. Rawlinson, L. Featherstone, M. Pitt, L. Caly, J. Druce, D. Purcell, L. Harty, T. Tran, J. Roberts, M. Catton, D. Williamson, L. Coin and S. Duchene (2020). "Direct RNA sequencing and early evolution of SARS-CoV-2." bioRxiv: 2020.2003.2005.976167.

Tyson, J. R., P. James, D. Stoddart, N. Sparks, A. Wickenhagen, G. Hall, J. H. Choi, H. Lapointe, K. Kamelian, A. D. Smith, N. Prystajecky, I. Goodfellow, S. J. Wilson, R. Harrigan, T. P. Snutch, N. J. Loman and J. Quick (2020). "Improvements to the ARTIC multiplex PCR method for SARS-CoV-2 genome sequencing using nanopore." bioRxiv: 2020.2009.2004.283077.

V'Kovski, P., A. Kratzel, S. Steiner, H. Stalder and V. Thiel (2021). "Coronavirus biology and replication: implications for SARS-CoV-2." Nat Rev Microbiol 19(3): 155-170.

Weil, P. P., J. Hentschel, F. Schult, A. Pembaur, B. Ghebremedhin, O. Mboma, A. Heusch, A. C. Reuter, D. Muller, S. Wirth, M. Aydin, A. C. W. Jenke and J. Postberg (2021). "Combined RT-qPCR and pyrosequencing of a Spike glycoprotein polybasic cleavage motif can uncover pediatric SARSCoV-2 infections associated with heterogeneous presentation." Mol Cell Pediatr 8(1): 4. Winger, A. and T. Caspari (2021). "The Spike of Concern-The Novel Variants of SARS-CoV-2." Viruses 13(6). 
medRxiv preprint doi: https://doi.org/10.1101/2021.07.08.21260171; this version posted July 24, 2021. The copyright holder for this preprint (which was not certified by peer review) is the author/funder, who has granted medRxiv a license to display the preprint in perpetuity. It is made available under a CC-BY-NC 4.0 International license.

\section{Figures and Figure legends}

349

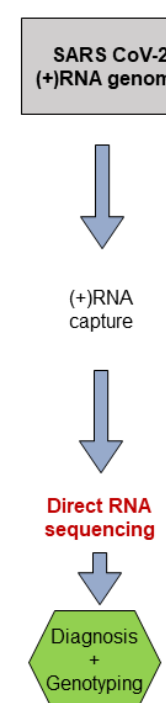

(2)
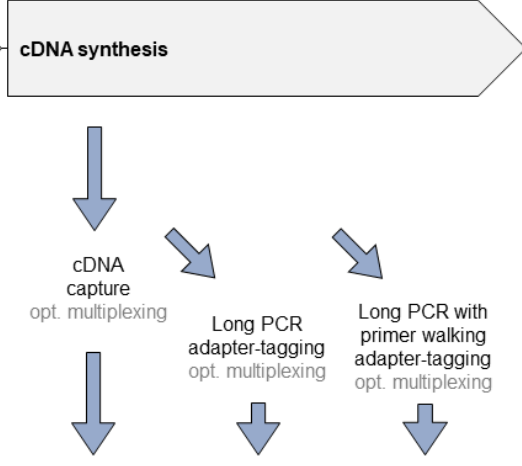

Direct cDNA
sequencing

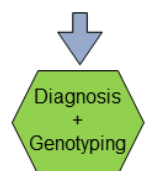

(3)

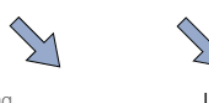

Long PCR adapter-tagging
opt. multiplexing

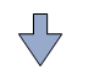

Long amplicon

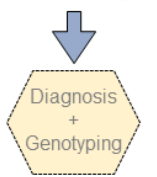

(4)

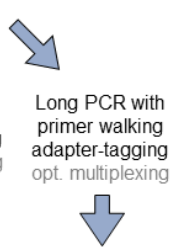

Long amplicon sequencing

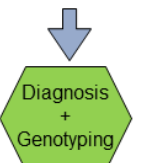

(5)
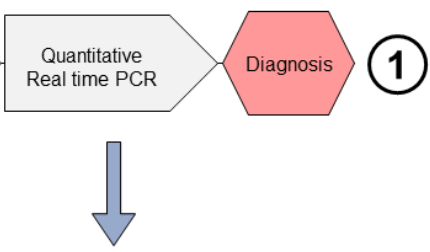

pyrosequencing or adapter-tagging opt. multiplexing

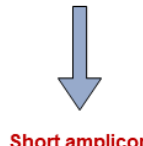

Short amplicon
sequencing

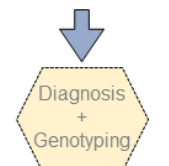

Partial genome coverage Full genome coverage

351 Figure 1. Nanopore sequencing options (2-6) compatible with diagnostic qPCR pipelines (1). The 352 pyrosequencing option in path (6) requires biotin-tagged primers for qPCR (Weil, Hentschel et al. 353 2021). 
medRxiv preprint doi: https://doi.org/10.1101/2021.07.08.21260171; this version posted July 24,2021 . The copyright holder for this preprint (which was not certified by peer review) is the author/funder, who has granted medRxiv a license to display the preprint in perpetuity. It is made available under a CC-BY-NC 4.0 International license.

A.
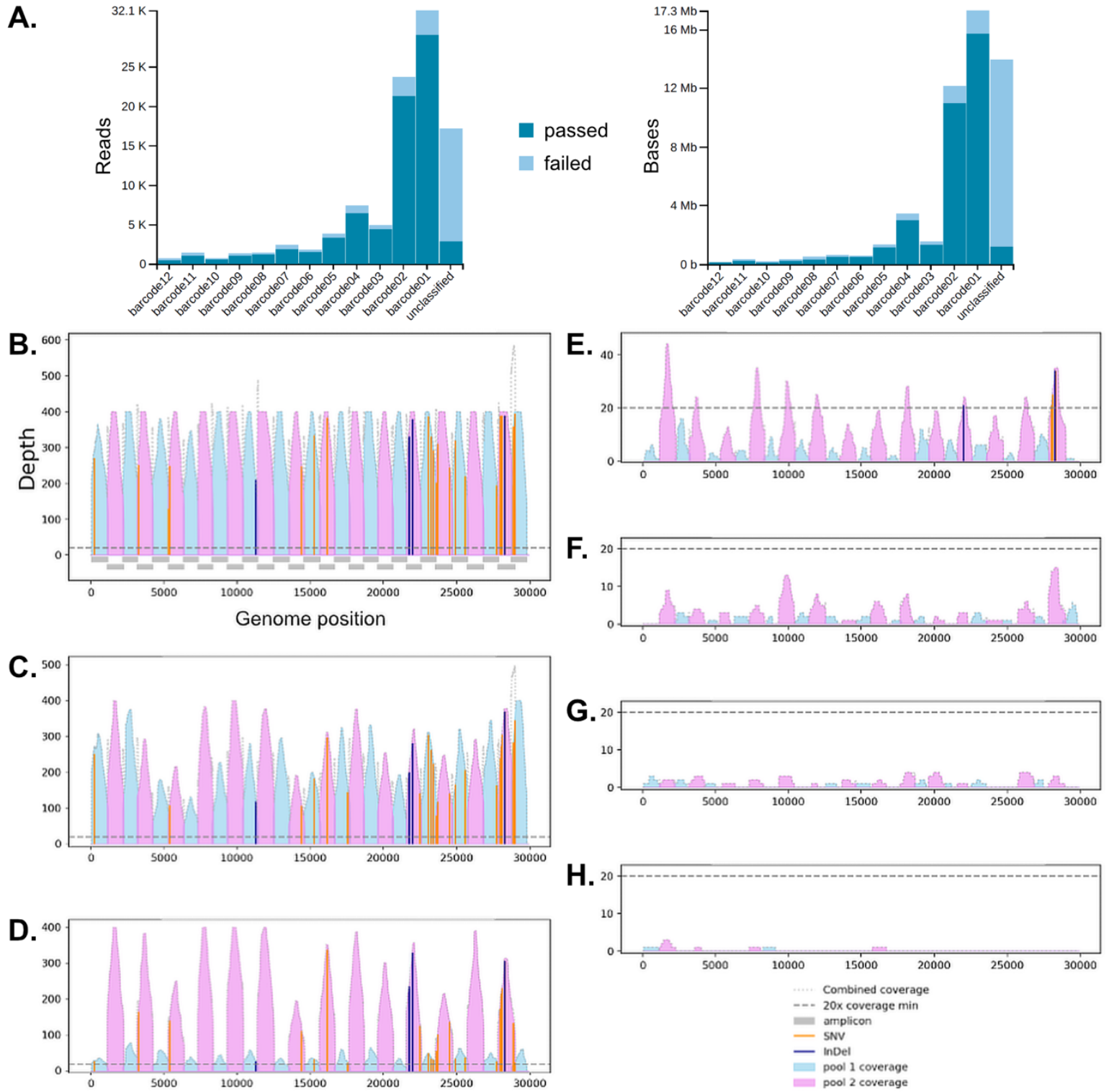

356 Figure 2. Surveillance using nanopore sequencing (A.), and effects of specimens dilution on the

357 SARS-CoV-2 genome coverage (B.), whereby viral copy numbers were $1.28 \times 10^{6}$ (B.); $5.12 \times 10^{8}$ (C.); $3582.56 \times 10^{8}$ (D.); $4 \times 10^{6}$ (E.); $2 \times 10^{6}$ (F.); $1 \times 10^{6}$ (G.); $5 \times 10^{5}$ (H.). In (A.) increasing barcode numbers 359 (X-axis) correspond to decreasing viral titers. 
medRxiv preprint doi: https://doi.org/10.1101/2021.07.08.21260171; this version posted July 24, 2021. The copyright holder for this preprint (which was not certified by peer review) is the author/funder, who has granted medRxiv a license to display the preprint in perpetuity. It is made available under a CC-BY-NC 4.0 International license.
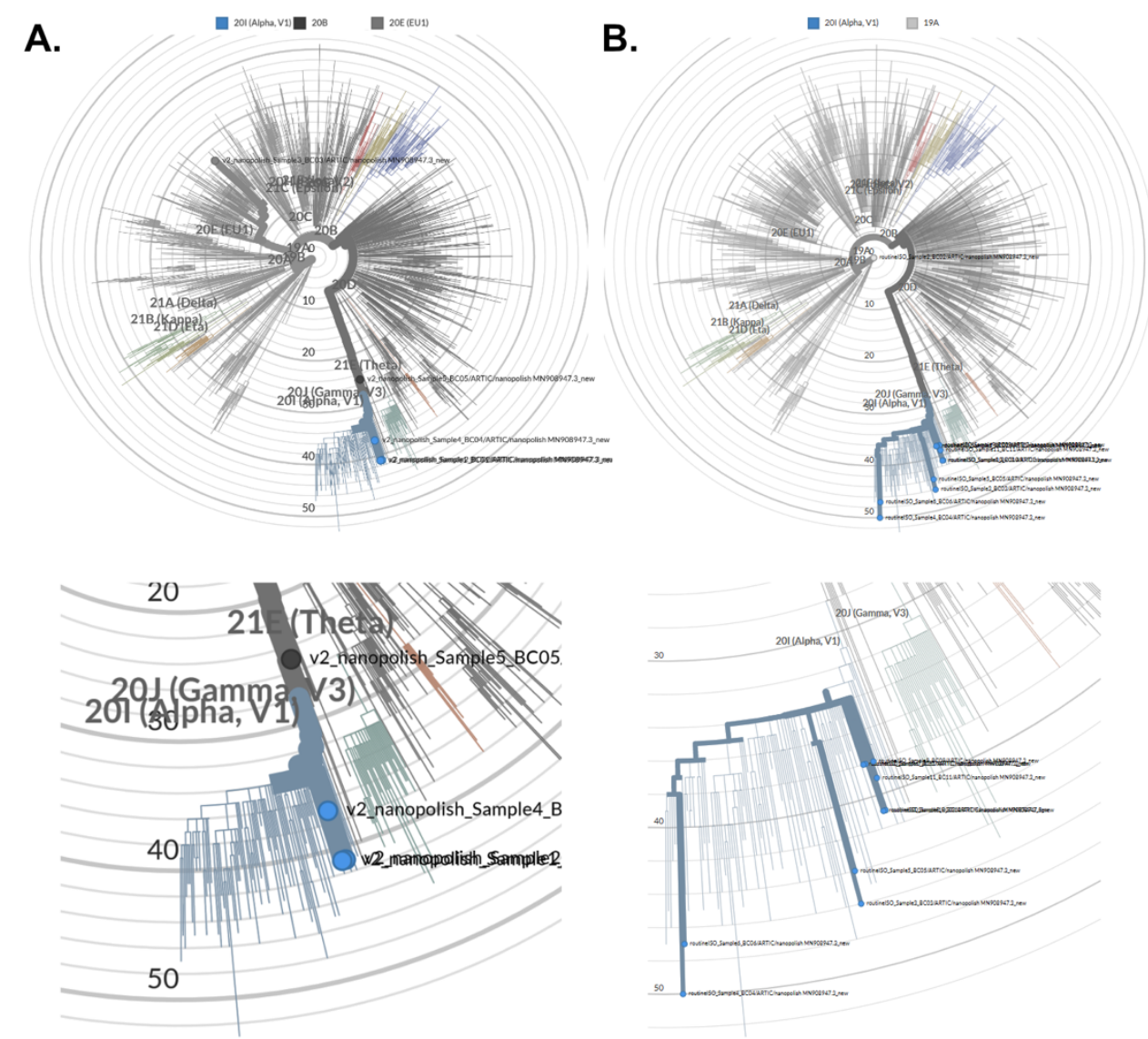

362 Figure 3. Comparison on the evolutionary distance calculation, when data from the same specimen

363 was processed by 2 different bioinformatics pipelines. Phylogenetic tree visualization was done using

364 the Nextstrain open-source platform for pathogen genome data analyses (Hadfield, Megill et al. 2018).

365 We used serial dilutions of identical specimens (A.), or a selection of samples from different

366 individuals from clinical routine (B.) for phylogenetic analyses. Below the trees a magnification of 367 clade 20I is shown, wherein most specimens grouped ( $\left.\mathrm{A}_{.,}, \mathrm{B}_{.}\right)$. 
medRxiv preprint doi: https://doi.org/10.1101/2021.07.08.21260171; this version posted July 24, 2021. The copyright holder for this preprint (which was not certified by peer review) is the author/funder, who has granted medRxiv a license to display the preprint in perpetuity. It is made available under a CC-BY-NC 4.0 International license.

369 Figure 4. Comparison of the consensus sequences returned by 'Nanopolish' and 'Medaka' algorithm 370 and the merged consensus for a nanopore sequencing run corresponding to a viral load of $1.28 \times 10^{* *} 6$.

A.

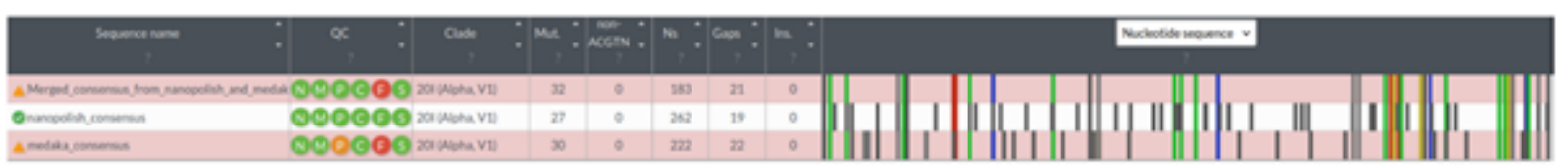

B. Label
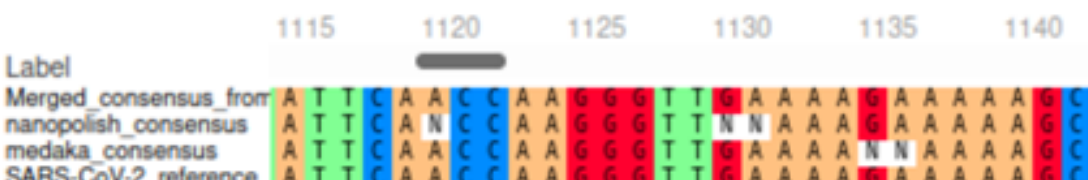

T T G A A A A GIA A A A A G C

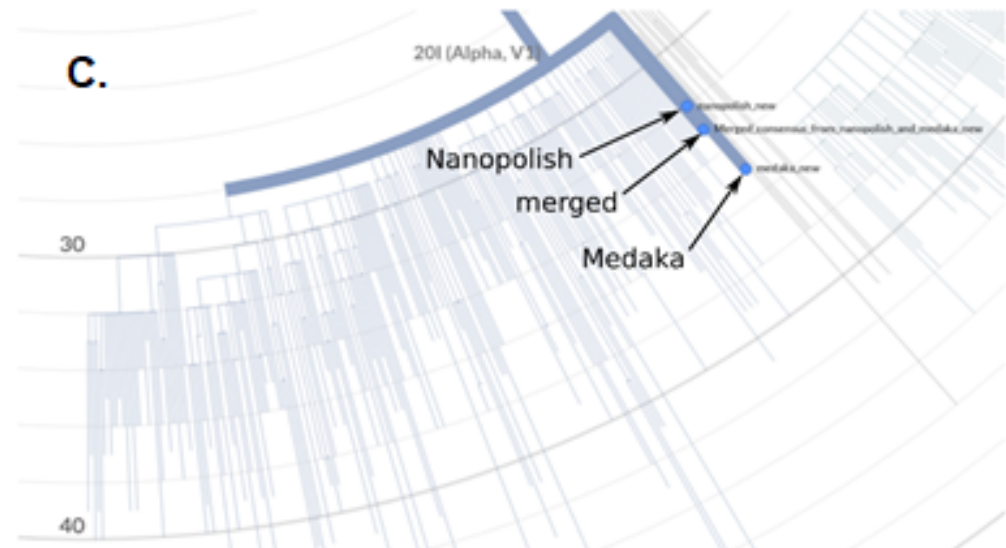

371 (A.) Alignment of the 'Nanopolish' and 'Medaka' consensus as well as the merged consensus on the

372 SARS-CoV-2 reference genome by the Nextstrain program. The merged consensus conserved all of

373 the high quality mutations that mapped to the known variant 20I (shown in colour or light grey), while

374 most non-matching mutations (in dark grey, likely sequencing errors) of the 'Nanopolish' or 'Medaka'

375 consensus were lost. (B.) Comparison of variant solving by 'Nanopolish', 'Medaka' and our code. The

376 multiple alignment was performed by MAFFT online tool with the three consensus sequences and the

377 SARS-CoV-2 reference genome (MN908947.3). Contrary to the 'Nanopolish' and 'Medaka'

378 consensus, the merged consensus solved the entire region and led to accurate variant calling. (C.)

379 Clade mapping and phylogenetic distances calculated by Nextstrain for the three consensus sequences. 
medRxiv preprint doi: https://doi.org/10.1101/2021.07.08.21260171; this version posted July 24, 2021. The copyright holder for this preprint (which was not certified by peer review) is the author/funder, who has granted medRxiv a license to display the preprint in perpetuity.

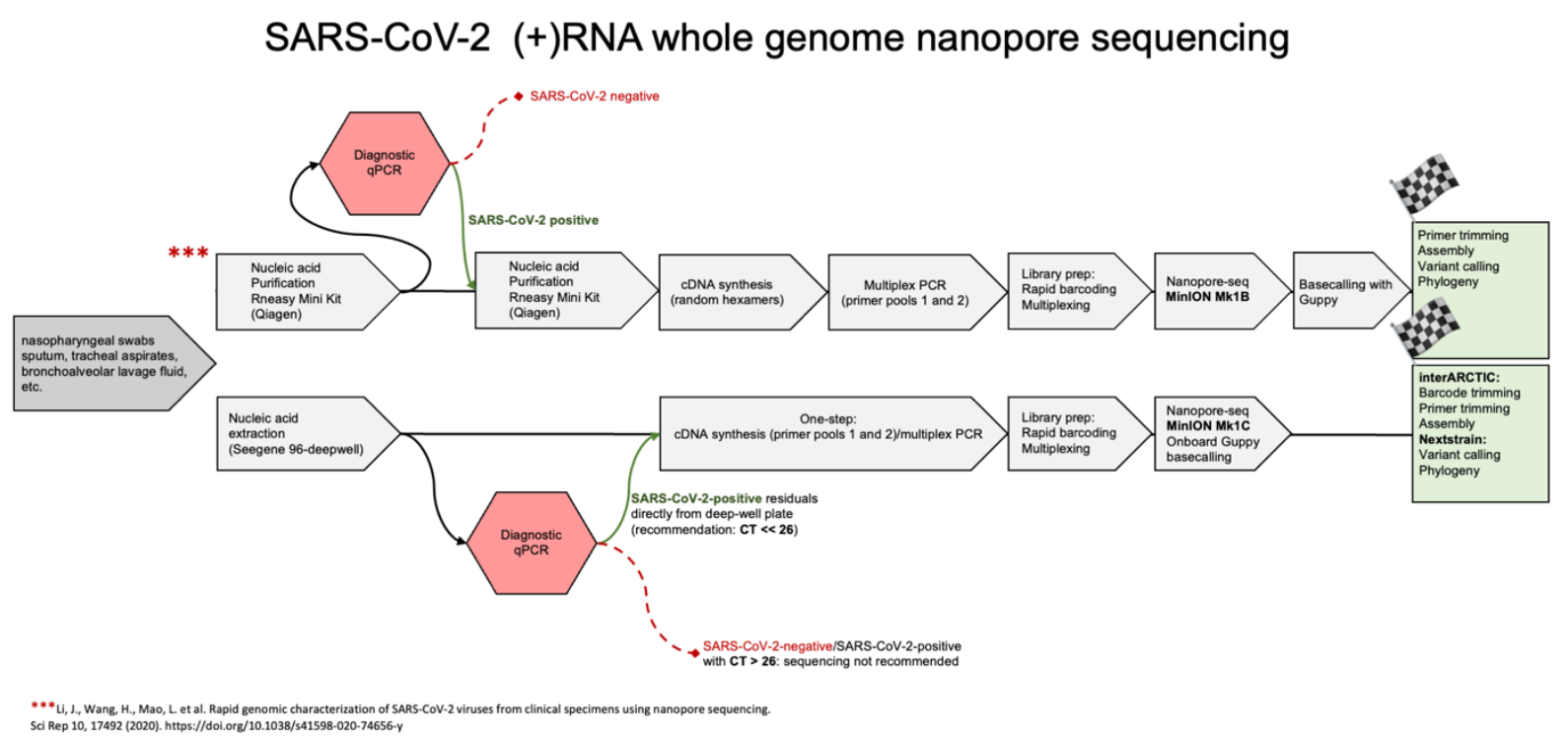

382 Figure 5. Comparison of a rapid SARS-CoV-2 whole (+)RNA genome nanopore sequencing pipeline

383 (Freed, Vlkova et al. 2020) with the novel simplified workflow, whose main achievements are: 1.

384 Purified RNA from SARS-CoV-2-positive patients can be directly taken from residual diagnostic

385 specimens in 96-deepwell-plates; 2. cDNA synthesis and successive multiplex PCR with 2 primer

386 pools can be performed in single tube reactions. Since cDNA synthesis is primed by SARS-CoV-2-

387 specific primers for $1,200 \mathrm{bp}$ amplicon amplification, there is no need for use of unspecific

388 hexanucleotide priming. 3. Onboard Guppy basecalling with the Oxford Nanopore MinION Mk1C

389 device and implementation of the interARTIC led to a further reduction of working steps and hands-on

390 time. 